\title{
ANÁLISIS DE LA NORMATIVIDAD EN SEGURIDAD Y SALUD OCUPACIONAL EN MINERÍA ENTRE LOS AÑOS 2000 Y 2017 Y SU INFLUENCIA EN LA OCURRENCIA DE ACCIDENTES MORTALES EN LA MINERÍA DEL PERÚ
}

\author{
ANALYSIS OF OCCUPATIONAL SAFETY AND HEALTH REGULATIONS IN \\ MINING BETWEEN 2000 AND 2017 AND ITS INFLUENCE ON THE \\ OCCURRENCE OF FATAL ACCIDENTS IN MINING IN PERU
}

\section{Jorge Luis Tomas Florez Salas}

\section{INTRODUCCIÓN}

En la actualidad existe en nuestro país, el Organismo Supervisor de la Inversión en Minería y Energía (OSINERGMIN) que tiene como ámbito de competencia "Supervisar y fiscalizar el cumplimiento de las disposiciones técnicas y legales de seguridad minera en la mediana y gran minería"; dicha supervisión y fiscalización la realiza conforme a lo establecido en el numeral 12.1 del artículo $12^{\circ} \mathrm{del}$ Reglamento de Supervisión y Fiscalización de las Actividades Energéticas y Mineras, aprobado por Resolución N 171-2013-OS/CD. La supervisión que realiza OSINERGMIN de las actividades mineras se divide en los siguientes temas: Ventilación en minería subterránea, Geo mecánica, Plantas de Beneficio, fundiciones, refinerías, depósitos de concentrado de mineral y plantas de relleno hidráulico, transporte, maquinarias e instalaciones auxiliares y depósito de relaves, pilas de lixiviación, depósitos de desmonte y tajo abierto; además de la investigación de la ocurrencia de los accidentes mortales en cuanto a las condiciones de seguridad en que ocurrió dicho suceso; todo ello enmarcado en la normatividad vigente en el Reglamento de Seguridad y Salud ocupacional (DS024-2016-EM y su reciente modificatoria, el DS-0232017-EM).

La accidentalidad mortal en el sector minero, dentro del ámbito de la mediana y gran minería, se encuentra cuantificada en los consolidados anuales que reportan los titulares de la actividad minera cuando tienen una ocurrencia de accidentes mortales, y que están a disposición de los usuarios del sector al publicarse en la web institucional tanto del MINEM y del OSINERGMIN; además dichas ocurrencias se encuentran también individualizadas, consignándose parámetros de Fecha de ocurrencia, Titular minero, Concesión/UEA, Nombre de la víctima, Empresa, Tipo de empresa a la que pertenece y Clasificación según Tipo. En términos generales la accidentalidad mortal en el sector minero, tanto en la mediana y gran minería, es un problema que está muy lejos de ser considerado en vías de solución; por el contrario, la tendencia va en aumento desde el año 2015 hasta lo que va del presente año.

\footnotetext{
${ }^{1}$ Ingeniero afiliado a la Escuela Profesional de Ingeniería de Minas de la Universidad Nacional de Moquegua (UNAM).
} 


\section{DESARROLLOTEMÁTICO}

Para el desarrollo de la presente investigación se usó el esquema de trabajo detallado en la Figura 1, el cual comprende el análisis de la normatividad vigente teniendo en cuenta la ocurrencia de accidentes mortales en dicho periodo de tiempo. Este análisis se realizó conformando tres grupos de trabajo que analizaron toda la información en jornadas de trabajo de 2 a 3 horas por semana, durante 2 meses, todo esto bajo la supervisión del director de investigación. Las jornadas se caracterizaron por ser sesiones de intercambio de ideas y debate acerca de la naturaleza de la ocurrencia de los accidentes mortales, para ello se tomó como base los reportes de ocurrencia de accidentes mortales desde el año 2002 hasta octubre de 2017 (el Anexo N ${ }^{\circ} 3$ es digital y agrupa todos los reportes de accidentes mortales desde el año 2002 hasta octubre de 2017), los mismos que se encuentran publicados en el portal web del Ministerio de Energía y Minas, según norma vigente.

Es necesario mencionar que también se hizo una nueva agrupación de los accidentes mortales, tomando como referencia el ciclo de minado en la minería, separados de los accidentes ocurridos en el proceso de beneficio de mineral y acumulación de material en desmonteras y relaveras. Como ya se mencionó en el párrafo anterior, en este proceso de agrupación se tomó como referencia el aviso de ocurrencia de accidente mortal a cargo del titular de actividad minera; el mismo que tiene por característica esencial resumir en dicho formato los aspectos más relevantes en que ocurrió el acontecimiento.

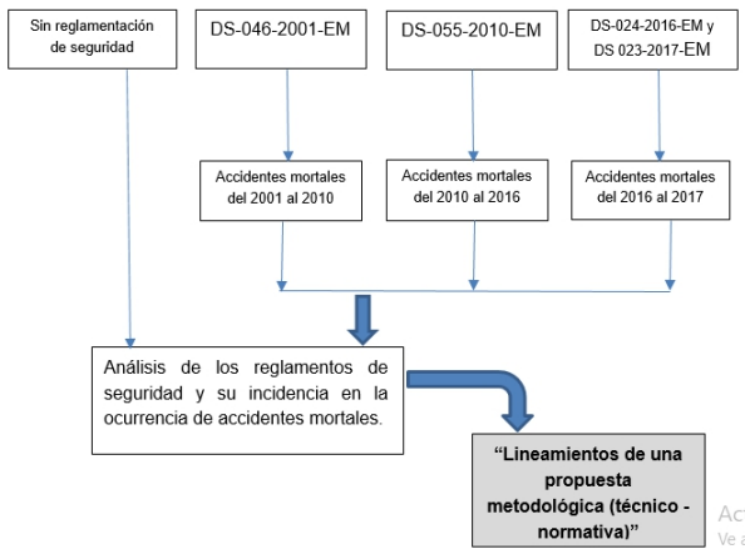

Figura 1. Esquema de trabajo

El resultado de las evaluaciones de los reportes de accidentes mortales según tipo notificados por los titulares de actividad minera, durante el periodo comprendido entre el año 2000 y noviembre de 2017 , buscan sentar las bases para la elaboración del futuro reglamento de seguridad y salud ocupacional que sustituya al vigente. El esquema de trabajo que persigue este fin se encuentra en la siguiente figura:

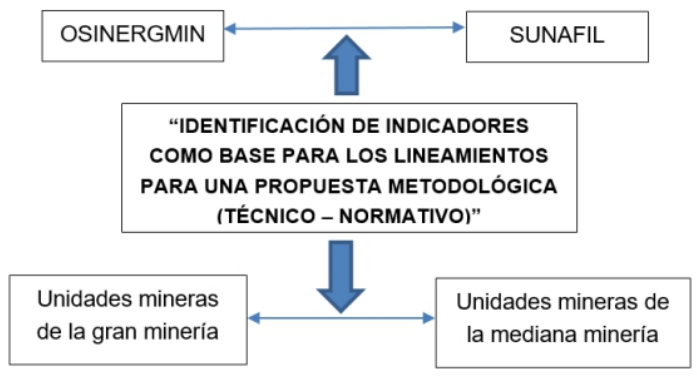

Figura 2. Estructura operativa

El resultado de la agrupación de la accidentalidad mortal desde el año 2000 a octubre de 2017 se estructuró según el modelo que se muestra en la Tabla $\mathrm{N}^{\circ} 1$; es necesario señalar que la penúltima columna, denominada "Otros", obedece a que los grupos de trabajo no pudieron establecer cuáles fueron las condiciones de ocurrencia del accidente, ya sea porque simplemente no se detalla nada o porque los datos son muy generales o imprecisos; cualquiera fuese la razón que tuvieron los titulares mineros a la hora de redactar la ocurrencia de los accidentes mortales, en su momento no ofrecieron la información convincente y necesaria para poderla catalogar dentro de las columnas anteriores del cuadro mencionado.

Una vez que se completó la revisión de toda la información disponible - relacionados con los títulos de gestión de operaciones mineras y servicios auxiliares y actividades conexas, así como las obligaciones de los titulares y supervisores del titular minero, todo ello enmarcado en las normas de seguridad que estuvieron en vigencia desde el año 2001 hasta el presente-, se procedió a valorar nuevamente los reportes de accidentes mortales que hicieron cada uno de los titulares de actividad minera durante todo ese periodo de tiempo, pero esta vez tomando en cuenta nuevos indicadores de accidentalidad generados por el suscrito, es decir, aspectos más relevantes acerca de la naturaleza de la ocurrencia de los accidentes mortales; no obstante, es necesario resaltar que dichos reportes son información preliminar acerca de la ocurrencia del mismo, por lo tanto aún sigue siendo información susceptible de ser cambiada durante la investigación de cada accidente mortal.

En las tablas 1, 2 y 3 se volvieron a evaluar los 974 accidentes mortales ocurridos desde el 01/01/2000 hasta el $31 / 10 / 2017$, clasificados en función a las actividades que conforman el ciclo de minado, separadas estas de las otras actividades posteriores que tienen sus propios estándares operacionales y procedimientos escritos de trabajo seguro. Además, como antes se mencionó, la columna "Otros" hace referencia a todos los accidentes con información insuficiente, por tanto, este aspecto constituye una limitación importante detectada durante el desarrollo de la presente investigación. 
Tabla 1. Clasificación de accidentes mortales del año 2000 hasta noviembre de 2017, en función de las actividades correspondientes a la gestión de operaciones mineras y gestión de servicios y actividades conexas.

\begin{tabular}{|c|c|c|c|c|c|c|c|c|}
\hline Periodo de vigencia & $\begin{array}{c}\text { Desate y } \\
\text { sostenimiento }\end{array}$ & $\begin{array}{c}\text { Perforación } \\
\text { y voladura }\end{array}$ & Ventilación & $\begin{array}{c}\text { Transporte } \\
\text { y acarreo }\end{array}$ & $\begin{array}{c}\text { Planta de } \\
\text { beneficio }\end{array}$ & Ralaveras & $\begin{array}{c}\text { Servicios y } \\
\text { actividades conexas }\end{array}$ & $\begin{array}{c}\text { Otros } \\
\text { TOTAL }\end{array}$ \\
\hline $\begin{array}{c}\text { Del 01-01-2000 hasta 24- } \\
\text { 07-2001. }\end{array}$ & 42 & 6 & 1 & 17 & - & -18 & 92 \\
\hline $\begin{array}{c}\text { Del 25-07-2001 al 21-08- } \\
\text { 10 (DS-046-2001-EM). }\end{array}$ & 174 & 66 & 58 & 79 & 19 & 7 & 72 \\
\hline $\begin{array}{c}\text { Del 22-08-2010 al 27-07- } \\
\text { 16 (DS-055-2010-EM). }\end{array}$ & 65 & 17 & 19 & 44 & 15 & 5 & 577 \\
\hline $\begin{array}{c}\text { Del 28-07-2016 hasta la } \\
\text { fecha (DS-024-2016-EM } \\
\text { y DS-023-2017-EM). }\end{array}$ & 11 & 1 & 3 & 6 & 4 & 2 & 102 \\
\hline TOTAL & 292 & 90 & 81 & 146 & 38 & 14 & 52 \\
\hline
\end{tabular}

\section{Consideraciones}

-Desate y sostenimiento: Cuantificar todos los acontecimientos ocurridos durante la etapa de desate y sostenimiento de labores, de manera manual y/o con equipos de sostenimiento.

- Perforación y voladura: Cuantificar todos los acontecimientos ocurridos durante la etapa de perforación y carga de frentes para su posterior disparo, además considerar explosión de tiros cortados y soplados.

-Ventilación: Cuantificar todos los acontecimientos ocurridos por inhalación de gases venenosos en interior mina, pero teniendo en cuenta que estén perfectamente señalados en el reporte de ocurrencia de accidente mortal.

- Transporte y acarreo: Cuantificar todos los acontecimientos ocurridos durante la etapa de acarreo y transporte de mineral y desmonte, este aspecto considera atropellos de las víctimas mortales, choque de vehículos y/o equipos, además de volcaduras y despiste de los mismos.

- Planta de beneficio: Cuantificar todos los acontecimientos ocurridos en plantas metalúrgicas, hidrometalúrgicas, electrometalúrgicas, piro metalúrgicas, etc. en cualquiera de sus etapas de proceso.

-Relaveras: Cuantificar todos los acontecimientos ocurridos en el área de influencia de las relaveras, escombreras y botaderos.

-Servicios y actividades conexas: Cuantificar todos los acontecimientos ocurridos durante la ejecución de actividades de electrificación, sistema de bombeo, mantenimiento mecánico, aire comprimido y sistemas de relleno en mina subterránea.

- Otros: Cuantificar todos los acontecimientos ocurridos fuera del ámbito de los parámetros anteriores.

Tabla 2. Clasificación de accidentes mortales año por año desde 2000 hasta octubre de 2017

\begin{tabular}{|c|c|c|c|c|c|c|c|c|c|}
\hline AÑo & $\begin{array}{c}\text { Desate y } \\
\text { sostenimiento }\end{array}$ & $\begin{array}{c}\text { Perforación } \\
\text { y voladura }\end{array}$ & Ventilación & $\begin{array}{c}\text { Transporte } \\
\text { y acarreo }\end{array}$ & $\begin{array}{l}\text { Planta de } \\
\text { beneficio }\end{array}$ & Ralaveras & $\begin{array}{c}\text { Servicios y } \\
\text { actividades conexas }\end{array}$ & Otros & TOTAI \\
\hline 2000 & 22 & 6 & 1 & 12 & - & - & $\mathbf{4}$ & 9 & 54 \\
\hline 2001 & 32 & 1 & - & 10 & - & - & 6 & 17 & 66 \\
\hline 2002 & 25 & 11 & 8 & 6 & 5 & 1 & 8 & 10 & 74 \\
\hline 2003 & 21 & 6 & 4 & 3 & 3 & - & 8 & 9 & 54 \\
\hline 2004 & 12 & 10 & 1 & 14 & 4 & - & 4 & 11 & 56 \\
\hline 2005 & 28 & 9 & 2 & 7 & - & - & 10 & 13 & 69 \\
\hline 2006 & 17 & 8 & 2 & 10 & 2 & 1 & 9 & 16 & 65 \\
\hline 2007 & 13 & 8 & 11 & 6 & 1 & - & 13 & 10 & 62 \\
\hline 2008 & 21 & 5 & 9 & 12 & 1 & 5 & 6 & 5 & 64 \\
\hline 2009 & 17 & 6 & 6 & 10 & 2 & - & 6 & 9 & 56 \\
\hline 2010 & 12 & 3 & 15 & 10 & 2 & - & 10 & 14 & 66 \\
\hline 2011 & 16 & 3 & 9 & 6 & 1 & - & 9 & 9 & 53 \\
\hline 2012 & 8 & 3 & 3 & 8 & 5 & 3 & 13 & 11 & 54 \\
\hline 2013 & 12 & 4 & 3 & 7 & 2 & 1 & 8 & 10 & 47 \\
\hline 2014 & 9 & 3 & 1 & 5 & 2 & - & 3 & 9 & 32 \\
\hline 2015 & 12 & 2 & 1 & 6 & 2 & 1 & 2 & 3 & 29 \\
\hline 2016 & 6 & 1 & 3 & 12 & 2 & 2 & 1 & 7 & 34 \\
\hline 2017 & 9 & 1 & 2 & 2 & 4 & - & 11 & 10 & 39 \\
\hline TOTAL & 292 & 90 & 81 & 146 & 38 & 14 & 131 & 182 & 974 \\
\hline
\end{tabular}


Análisis de la normatividad en seguridad y salud ocupacional en minería entre los años 2000 y 2017 y su influencia en la ocurrencia de accidentes mortales en la minería del Perú

Julio - diciembre 2018

Tabla 3. Clasificación de accidentes mortales de los años 2001, 2010 y 2016 donde se presentaron los correspondientes cambios de la norma

\begin{tabular}{|c|c|c|c|c|c|c|c|c|c|}
\hline $\begin{array}{c}\text { NORMA } \\
\text { Periodo de vigencia }\end{array}$ & $\begin{array}{c}\text { Desate y } \\
\text { sostenimiento }\end{array}$ & $\begin{array}{c}\text { Perforación } \\
\text { y voladura }\end{array}$ & Ventilación & $\begin{array}{c}\text { Transporte } \\
\text { y acarreo }\end{array}$ & $\begin{array}{l}\text { Planta de } \\
\text { beneficio }\end{array}$ & Relaveras & $\begin{array}{c}\text { Servicios } y \\
\text { actividades conexas }\end{array}$ & Otros & TOTAL \\
\hline $\begin{array}{c}\text { Norma Ausente } \\
\text { Del 01-01-2001 al 24-07-2001 }\end{array}$ & 20 & - & - & 5 & - & - & 4 & 9 & 38 \\
\hline $\begin{array}{c}\text { DS-046-2001 } \\
\text { Del 25-07-2001 al 31-12-2001 }\end{array}$ & 12 & 1 & - & 5 & - & - & 2 & 8 & 28 \\
\hline $\begin{array}{c}\text { DS-046-2001 } \\
\text { Del 01-01-2010 al 21-08-2010 }\end{array}$ & 8 & 2 & 15 & 6 & 1 & - & 6 & 11 & 49 \\
\hline $\begin{array}{c}\text { DS-055-2010 } \\
\text { Del 22-08-2010 al 31-12-2010 }\end{array}$ & 4 & 1 & - & 4 & 1 & - & 4 & 3 & 17 \\
\hline $\begin{array}{c}\text { DS-055-2010 } \\
\text { Del 01-01-2016 al 27-07-2016 }\end{array}$ & 4 & 1 & 2 & 8 & 2 & - & - & 4 & 21 \\
\hline $\begin{array}{c}\text { DS-024-2016 } \\
\text { Del 28-07-2016 al 31-12-2016 }\end{array}$ & 2 & - & 1 & 4 & - & 2 & 1 & 3 & 13 \\
\hline TOTAL & 50 & 5 & 18 & 32 & 4 & 2 & 17 & 38 & 166 \\
\hline
\end{tabular}

\section{DISCUSIÓN}

En la revisión de cada uno de los capítulos, subcapítulos y artículos del título correspondiente a la gestión de las operaciones mineras y servicios, tanto de las normas derogadas como del reglamento de seguridad vigente y su modificatoria, no se aprecia una evolución de los diferentes artículos que tengan una clara postura preventiva sino, por el contrario, muestran un enfoque absolutamente restrictivo y prohibitivo en su gran mayoría, llegando incluso a la exageración en ciertos casos donde algunos artículos son repetidos exactamente igual de una norma a otra; en consecuencia, es necesario que en la futura norma de seguridad se precise con mayor claridad las condiciones más seguras para la realización de los trabajos en las unidades mineras.

Por otro lado, se verificó que en el análisis de los reportes de accidentes mortales (ocurridos durante el año 2002 y octubre de 2017), 2/3 de los accidentes se suscitaron en actividades tipificadas de alto riesgo; por lo tanto, existe $1 / 3$ de ocurrencia de accidentes mortales fuera de esta tipificación de "alto riesgo". Es necesario tomar en cuenta este indicador para que dichas actividades tengan un mejor y mayor control por parte de los titulares mineros y los organismos supervisores.

Se estableció con claridad que la normatividad, durante este periodo, no estuvo enmarcada en proteger al trabajador en el ciclo de minado de forma explícita; antes bien, se revela un incremento de artículos, subcapítulos, capítulos y hasta un título más que establece nuevas actividades dentro del proceso productivo. Lo cual no está mal, sin embargo, demuestra un señalamiento hacia la incorporación de actividades y/o procesos nuevos en el sector minero, dejando en segundo plano la protección del trabajador.

Así también, se realizó el análisis en relación a cada una de las hipótesis planteadas en el proyecto de investigación, lo que se detalla a continuación:

Si algo tienen en común las normas de seguridad es que no precisan, con mayor claridad, las mejores condiciones de seguridad para la realización de los trabajos en las unidades mineras. En las tablas 1, 2 y 3 se observa una tendencia en la reducción de accidentes mortales, comparando la década anterior con respecto a lo que va de la presente década, se concluye que dichos accidentes en los primeros años de este siglo están por encima de 50 en promedio, contrastado con los 50 por debajo que se mantiene en la década actual; más grave aún, durante los 3 últimos años existe una evidente tendencia en aumento, como se muestra en la figura 3. En tal sentido la hipótesis general ha quedado demostrada en el análisis realizado.

Es importante señalar que en la revisión de los 974 accidentes mortales ocurridos entre el año 2000 y 2017, 2/3 de ellos responden aproximadamente a condiciones de alto riesgo; esto significa que $1 / 3$ de accidentes ocurrieron en otras actividades. A partir de este dato, se pone de manifiesto que la peligrosidad de la actividad minera no solamente se circunscribe a las actividades de alto riesgo.

Finalmente, a través del análisis de la parte normativa, así como de los reportes de ocurrencia de accidentes mortales, se advierte que en los más de 400 artículos que actualmente tiene la norma vigente no se precisan mejores aspectos de seguridad en las diferentes etapas de minado que se contemplan en dicha norma. 


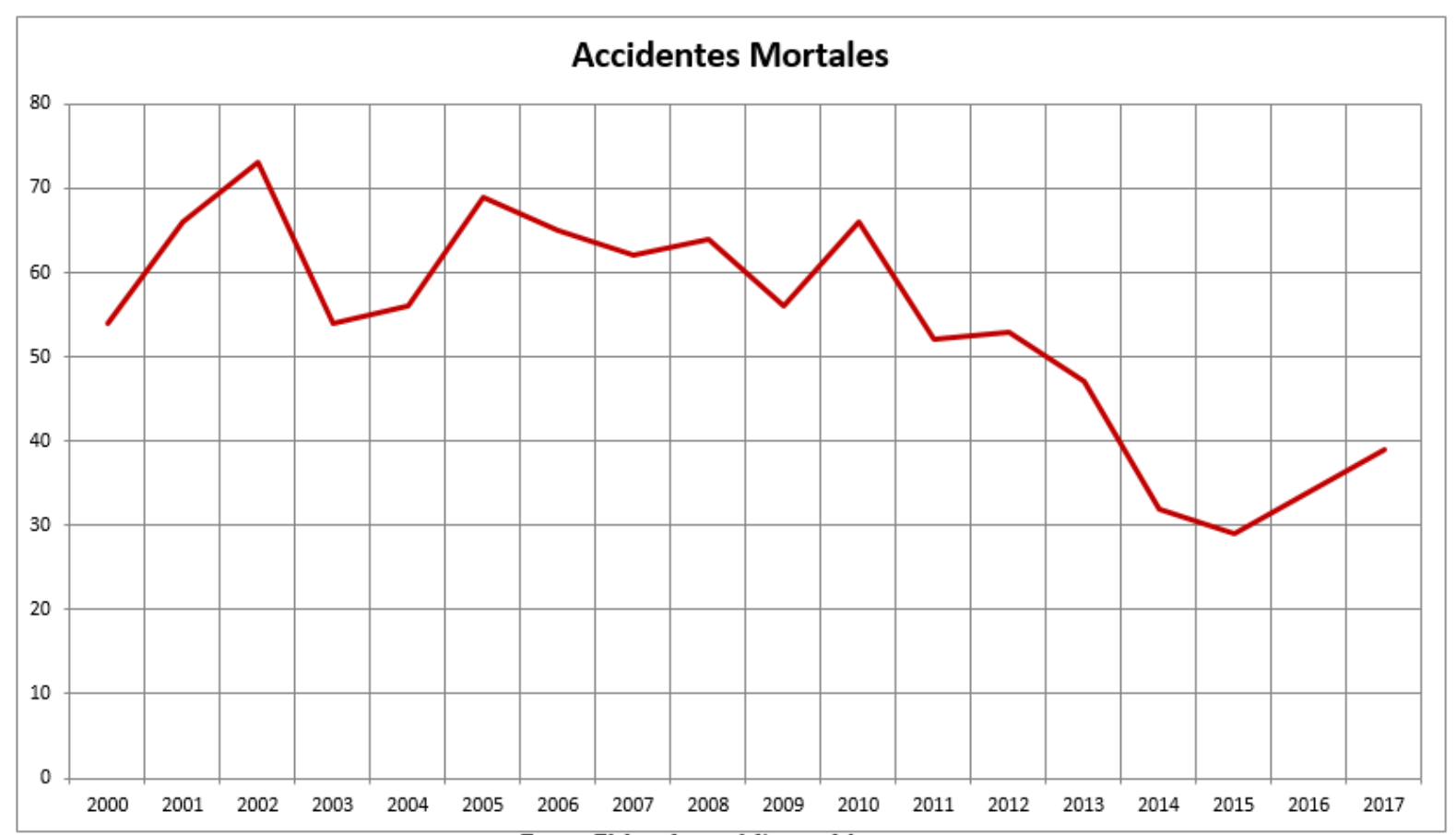

Figura 3. Evolución de los accidentes mortales desde el año 2000 hasta octubre del año 2017

\section{CONCLUSIONES}

Las normas de seguridad y salud ocupacional vigentes durante el periodo de tiempo comprendido entre el año 2000 y noviembre de 2017 no precisan con claridad las mejores condiciones de seguridad para la realización de los trabajos dentro de las unidades mineras; por lo tanto, no contribuyen a que descienda de forma progresiva y sustancial la tasa de ocurrencia de accidentes mortales en la gran, mediana y pequeña minería, así como en la minería artesanal.

La ocurrencia de accidentes mortales en la mediana y gran minería no se dio en las actividades tipificadas de alto riesgo por el Reglamento de Seguridad y Salud Ocupacional vigente desde el año 2000 hasta noviembre de 2017.

Los diferentes títulos, capítulos y subcapítulos de los reglamentos de seguridad y salud ocupacional que tuvieron vigencia desde el año 2001 hasta 2017 han incrementado el número de artículos en función a la tipificación de nuevas actividades dentro del ámbito de la mediana y gran minería, mas no en precisar mejores aspectos de seguridad de las diferentes etapas de minado que se contemplan en dichas normas.

\section{BIBLIOGRAFÍA CONSULTADA}

Ley General de Minería Texto Único Ordenado TUO DS No 014-92-EM (1992). Normas Legales del Perú.

Ley de Seguridad y Salud en el Trabajo - Ley No 29783 (2011)- Normas Legales del Perú.
Ley del Código de Ética de la Función Pública Ley N ${ }^{\circ}$ 27815 (2002). Normas Legales del Perú.

Ministerio de Energía y Minas - Reportes de Accidentes Mortales en Minería (2010). Recuperado de: http://www.minem.gob.pe.

Modificatoria de la Ley del Código de Ética de la Función Pública Ley $\mathrm{N}^{\circ} 28496$ (2005). Normas Legales del Perú.

Modificatoria del Reglamento de Seguridad y Salud Ocupacional DS No 023-2017-EM (2017). Normas Legales del Perú.

Modificatoria de la Ley de Seguridad y Salud en el Trabajo Ley $\mathrm{N}^{\circ} 30222$ (2014). Normas Legales del Perú.

Modificatoria del Reglamento de la Ley de Seguridad y Salud en el Trabajo DS Nº 006-2014-TR (2014). Normas Legales del Perú.

Plan Nacional de Seguridad y Salud en el Trabajo 2017 - 2021 DS N ${ }^{\circ}$ 005-2017-TR (2017). Normas Legales del Perú.

Picozi Bilbao, Aldo (2005). Mercado internacional del oro y minería del oro en Chile. Documento de Trabajo N ${ }^{\circ}$ 108. Comisión Chilena del Cobre (COCHILCO).

Política Nacional de Seguridad y Salud en el Trabajo $\mathrm{DS} \mathrm{N}^{\circ}$ 002-2013-TR (2013). Normas Legales del Perú.

OHSAS 2018:2007 Sistemas de Gestión de Seguridad y Salud en el Trabajo - Requisitos (2007).

OHSAS 18002:2008 Sistemas de Gestión de Seguridad y Salud en el Trabajo - Directrices para la implementación de OHSAS 2018:2007. 
Reglamento de Seguridad e Higiene Minera DS N 046-2001-EM (2001). Normas Legales del Perú.

Reglamento de Seguridad y Salud Ocupacional DS N ${ }^{\circ}$ 055-2010-EM (2010). Normas Legales del Perú.

Reglamento de Seguridad y Salud Ocupacional DS N ${ }^{\circ}$ 024-2016-EM (2016). Normas Legales del Perú.

Reglamento de la Ley de Seguridad y Salud en el Trabajo DS $\mathrm{N}^{\circ}$ 005-2012-TR (2012). Normas Legales del Perú.

Reglamento de la Ley del Código de Ética de la
Función Pública DS N 033-2005-PCM (2005). Normas Legales del Perú.

Vásquez, Arturo (2006). El Valor de la Vida Estadística y sus aplicaciones a la Fiscalización de la Industria de Hidrocarburos. Documento de Trabajo N ${ }^{\mathrm{o}} 18$. Oficina de Estudios Económicos OSINERGMIN.

Valega, José M. (1939). "El Virreinato del Perú". Editorial: Cultura Ecléctica. Lima-Perú. 